\title{
Aortic coarctation augments changes in thoracic aortic hemodynamics in pediatric and young adult patients with bicuspid aortic valve
}

\author{
Bradley D Allen ${ }^{1 *}$, Alex J Barker ${ }^{1}$, Maya Gabbour ${ }^{4}$, Michael Markl', Cynthia Rigsby ${ }^{4,1}$, Joshua D Robinson ${ }^{2,3}$ \\ From 16th Annual SCMR Scientific Sessions \\ San Francisco, CA, USA. 31 January - 3 February 2013
}

\section{Background}

Bicuspid aortic valve (BAV) is a congenital abnormality that is often associated with aortic coarctation. Evidence suggests this combination may increase the risk of secondary aortic complications compared to patients with BAV alone.[1] Altered aortic hemodynamics likely induce vascular remodeling[2] and may contribute to these complications. No study has investigated the impact of aortic coarctation on aortic hemodynamics in pediatric and young adult patients with BAV using time-resolved three-dimensional phase contrast (4D flow) MRI. The aim of this study is to utilize 4D flow MRI to compare thoracic aorta flow patterns in pediatric and young adult patients with BAV with and without aortic coarctation or coarctation repair.

\section{Methods}

In accordance with an IRB-approved protocol, 17 patients with BAV (age $=11.9+/-5.3$ years) who underwent cardiovascular MRI including 4D flow as part of follow-up care were included. Seven patients had aortic coarctation including 5 patients with coarctation repairs. Images were processed using in-house software for noise reduction, anti-aliasing, and eddy current correction. Flow visualization and quantification were performed in EnSight (CEI, Apex, NC). Aortic root z-scores were calculated from MRI measurements and height and weight at time of scan in EchoIMS (Merge, Chicago, IL). Flow jet pattern and helicity were assessed in the ascending aorta, aortic arch, and descending aorta. Flow jet pattern was determined by the region of peak velocity in systole corresponding to anatomically designated quadrants of

${ }^{1}$ Radiology, Northwestern University Feinberg School of Medicine, Chicago, IL, USA

Full list of author information is available at the end of the article

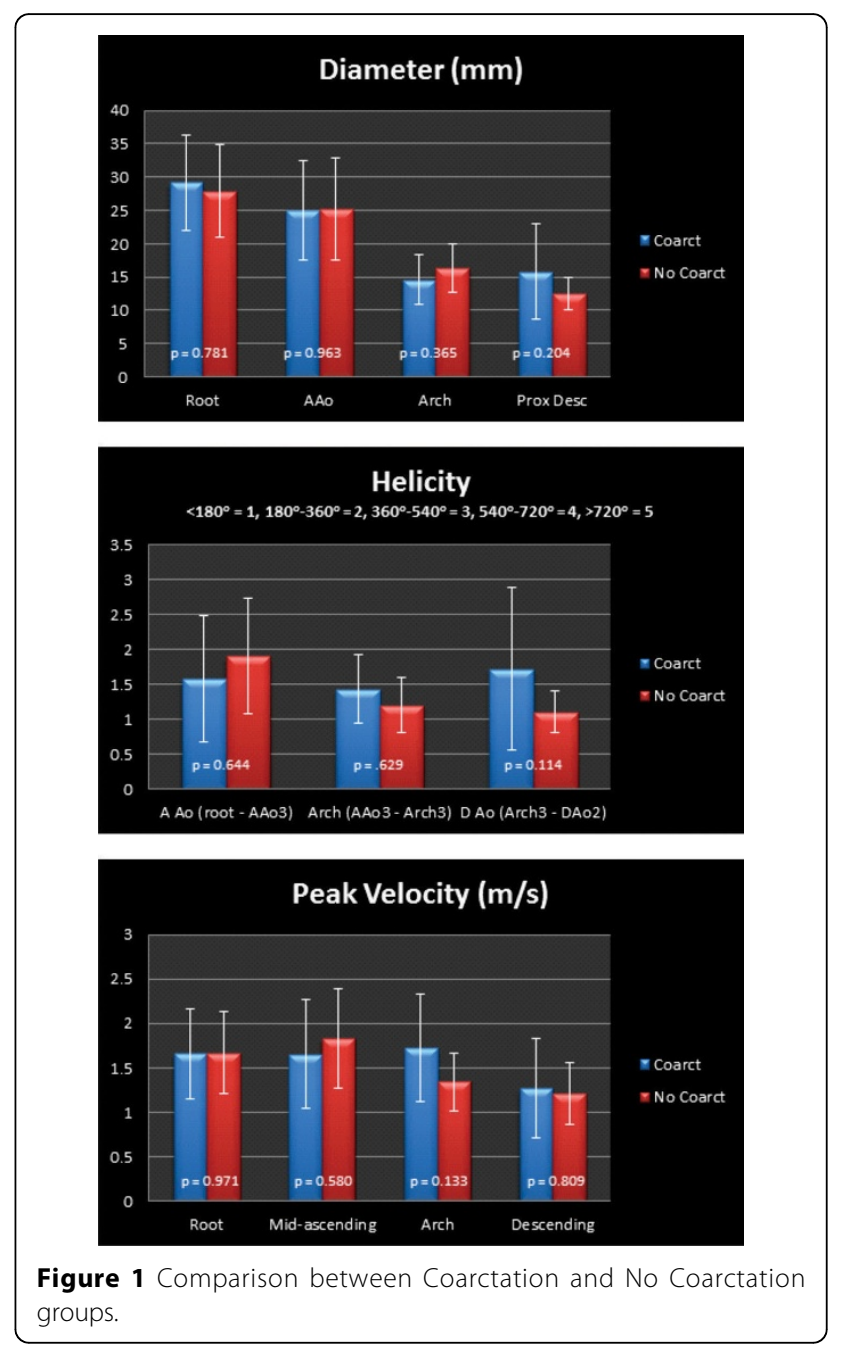

\footnotetext{
Fult ist of author information is available at the end of the article
}

(c) 2013 Allen et al; licensee BioMed Central Ltd. This is an Open Access article distributed under the terms of the Creative Commons B.1.: Central Attribution License (http://creativecommons.org/licenses/by/2.0), which permits unrestricted use, distribution, and reproduction in 


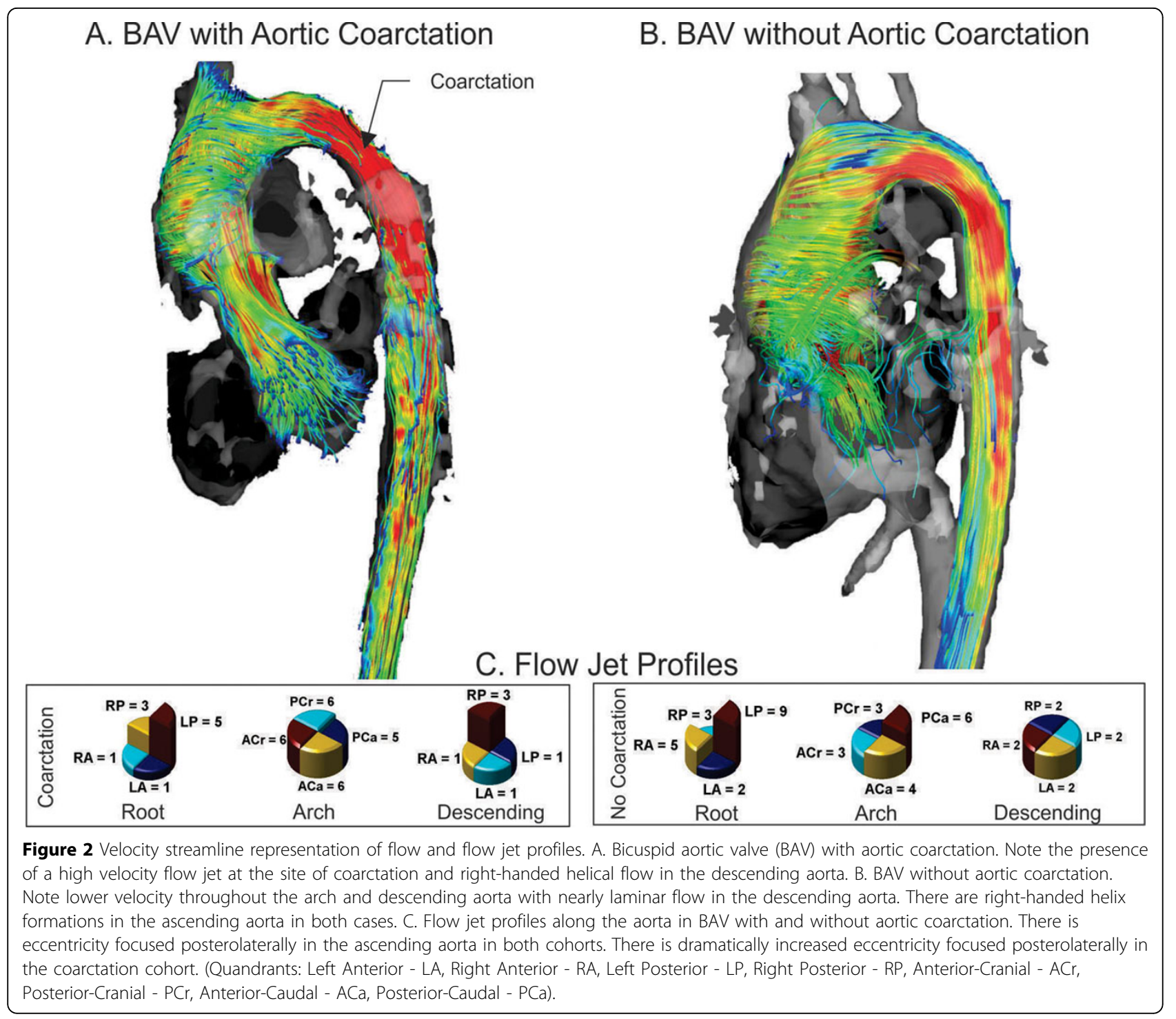

planes placed perpendicular to flow in each region of interest (ROI). Helicity was graded on a scale of $0-5$ based on the rotation angle within each ROI. Helicity was compared using a Wilcoxon rank-sum test. All other data were compared using a Student's t-test.

\section{Results}

There was no significant difference in the age $(13.2+/-6.5$ years vs. $11.0+/-4.1$ years, $\mathrm{p}=0.433$ ) or the root $\mathrm{z}$-scores $(2.8+/-1.4$ vs. $2.9+/-2.0, \mathrm{p}=.983)$ between the coarctation $(\mathrm{n}=7)$ and non-coarctation $(\mathrm{n}=10)$ groups. There was a trend toward higher arch velocities, increased helicity in the descending aorta, smaller arch diameter, and larger descending aorta diameter in the coarctation group, although no difference was statistically significant (Figure 1). There was a high degree of helicity in the ascending aorta in both groups. The coarctation group had more eccentric flow in the descending aorta (Figure 2).

\section{Conclusions}

In pediatric and young adult patients with BAV, aortic coarctation or coarctation repair is associated with a trend towards increased flow derangement relative to the non-coarctation group, but the power to detect a significant difference was limited by cohort size. Future work should focus on correlating outcomes such as aneurysm formation and re-operation with flow characteristics in this population.

\section{Funding}

Grant support: NIH R01HL115828, NUCATS Dixon Award. 


\section{Author details}

'Radiology, Northwestern University Feinberg School of Medicine, Chicago, IL, USA. ${ }^{2}$ Pediatrics, Northwestern University Feinberg School of Medicine, Chicago, IL, USA. ${ }^{3}$ Cardiology, Ann \& Robert H Lurie Children's Hospital of Chicago, Chicago, IL, USA. ${ }^{4}$ Medical Imaging, Ann \& Robert H Lurie

Children's Hospital of Chicago, Chicago, IL, USA.

Published: 30 January 2013

\section{References}

1. Oliver JM, et al:. Am J Cardiol 2009, 104:1001-1006.

2. Barker AJ, et al.. Circ Cardiovasc Imaging 2012, 5:457-466.

doi:10.1186/1532-429X-15-S1-P300

Cite this article as: Allen et al:: Aortic coarctation augments changes in thoracic aortic hemodynamics in pediatric and young adult patients with bicuspid aortic valve. Journal of Cardiovascular Magnetic Resonance 2013 15(Suppl 1):P300.

Submit your next manuscript to BioMed Central and take full advantage of:

- Convenient online submission

- Thorough peer review

- No space constraints or color figure charges

- Immediate publication on acceptance

- Inclusion in PubMed, CAS, Scopus and Google Scholar

- Research which is freely available for redistribution

Submit your manuscript at www.biomedcentral.com/submit
C Biomed Central 